\title{
Receptor-Like Tyrosine Phosphatase PTP10D Is Required for Long-Term Memory in Drosophila
}

\author{
Meng Qian, ${ }^{1 \star}$ Guohui Pan, ${ }^{1 \star}$ Lu Sun, ${ }^{1}$ Chunhua Feng, ${ }^{3}$ Zuoping Xie, ${ }^{1}$ Tim Tully, ${ }^{2}$ and Yi Zhong ${ }^{1,2}$ \\ ${ }^{1}$ Department of Biological Science and Biotechnology, Tsinghua University, Beijing, China 100084, ${ }^{2}$ Cold Spring Harbor Laboratory, Cold Spring Harbor, \\ New York, 11724, and ${ }^{3} J o e K a i$ Inc., Beijing, China 100084
}

Tyrosine phosphorylation mediates multiple signal transduction pathways that play key roles in developmental processes and behavioral plasticity. The level of tyrosine phosphorylation is regulated by protein tyrosine kinases and protein tyrosine phosphatases (PTPs). Extensive studies have investigated the roles of tyrosine kinases in memory formation. However, there were few studies on PTPs. To date, learning has been shown to be defective only for mouse knock-outs of PTP $\alpha$, leukocyte common antigen-related, or PTP $\delta$. A major limitation of these studies arises from their inability to distinguish an acute (biochemical) impairment of memory formation from a more chronic abnormality in neurodevelopment. From a behavioral screen for defective long-term memory, we found chi mutants to disrupt expression of the PTP10D protein tyrosine phosphatase gene. We show that chi mutants are normal for learning, early memory, and anesthesia-resistant memory, whereas long-term memory specifically is abolished. Significantly, induction of a heat shock-PTP10D ${ }^{+}$ transgene before training fully rescues the memory defect of chi mutants, thereby demonstrating an acute role for PTP10D in behavioral plasticity. We show that PTP10D is widely expressed in the embryonic CNS and in the adult brain. Transgenic expression of upstream activating sequence-PTP10D ${ }^{+}$in mushroom bodies is sufficient to rescue the memory defect of chi mutants. Our data clearly demonstrate that signaling through PTP10D in mushroom bodies is critical for the formation of long-term memory.

Key words: PTP10D; long-term memory; mushroom bodies; chi; behavior; screen

\section{Introduction}

Tyrosine phosphorylation mediates multiple signal transduction pathways that play key roles in developmental processes, as well as behavioral plasticity (Neel and Tonks, 1997; Van Vactor et al., 1998). The level of tyrosine phosphorylation is known to be regulated by protein tyrosine kinases and protein tyrosine phosphatases (PTPs) (Van Vactor et al., 1998). Extensive studies have investigated the roles of different families of tyrosine kinases in memory formation of vertebrates and invertebrates (for review, see Purcell and Carew, 2003). However, there were few studies on the involvement in memory formation of PTPs. To date, learning has been shown to be defective only for mouse knock-outs of $\operatorname{PTP} \alpha$, leukocyte common antigen-related, or PTP $\delta$ (Uetani et al., 2000; Skelton et al., 2003; Kolkman et al., 2004). A major limitation of these studies arises from their inability to distinguish an acute (biochemical) impairment of memory formation from a more chronic abnormality in neurodevelopment (Wallace et al., 1999; Uetani et al., 2000; Kolkman et al., 2004).

Received Sept. 16, 2006; revised Feb. 13, 2007; accepted Feb. 16, 2007.

This work was supported by grants from the National Natural Sciences Foundation of China (30220120692), the National Basic Research Program of China (973 Program, 2006CB500800), Tsinghua-Yue-Yuen Medical Sciences Fund (Y.Z.), and the Dart Foundation (T.T., Y.Z.). We are grateful to Kai Zinn and Developmental Studies Hybridoma Bank for providing antibodies to PTP10D, to Kai Zinn, Lai Zhichun, and the Bloomington stock center for providing stocks, and to Robert Saint and Berkeley Drosophila Genome Project for providing the plasmid re52018.

${ }^{*} M$.Q. and G.P. contributed equally to this work.

Correspondence should be addressed to Yi Zhong, Cold Spring Harbor Laboratory, P.0. Box 100, Cold Spring Harbor, NY 11724. E-mail: zhongyi@cshl.edu.

DOI:10.1523/JNEUROSCI.4054-06.2007

Copyright $\odot 2007$ Society for Neuroscience $\quad$ 0270-6474/07/274396-07\$15.00/0
Behavioral, pharmacological, and genetic experiments on memory formation after pavlovian olfactory learning in Drosophila have revealed four temporal phases: short-term memory (STM), middle-term memory (MTM), anesthesia-resistant memory (ARM), and long-term memory (LTM) (Tully et al., 1990). STM, MTM, and ARM are insensitive to cycloheximide (protein synthesis inhibitor) and are present after a single training session or after massed training (10 training sessions with no rest interval between each). LTM, in contrast, depends on protein synthesis and is uniquely induced by spaced training (10 training sessions with a 15 min rest interval between each). One day after spaced training, memory retention comprises approximately equal amounts of ARM and LTM, whereas $1 \mathrm{~d}$ memory after massed training comprises only ARM.

Based on a behavioral screen, we found PTP10D mutants to be defective in $1 \mathrm{~d}$ memory after spaced training. In Drosophila, PTP10D is one of the six receptor-like PTPs. (Schindelholz et al., 2001). PTP10D has a receptor-like extracellular region consisting of fibronectin type II domains, linked via a single transmembrane domain to a cytoplasmic region containing one phosphatase domain (Tian et al., 1991; Yang et al., 1991). Its extracellular structure is similar to that of the cell adhesion molecules, suggesting that it mediates interaction between cells. PTP10D single mutants are viable, fertile, and display no abnormal embryonic phenotypes (Desai et al., 1996; Sun et al., 2000). In PTP10D;PTP69D (encoding another PTP) double mutants, however, growth cone activity across the midline of the CNS is altered in embryos (Sun et al., 2000). 
Here, we demonstrate that PTP10D mutants are normal for learning, early memory, and anesthesia-resistant memory, whereas long-term memory specifically is abolished. We show that PTP10D is widely expressed in the embryonic CNS and in the adult brain. Moreover, induced expression of a heat shock (hs)-PTP10D ${ }^{+}$transgene in the adult and expression of upstream activating sequence (UAS)-PTP10D ${ }^{+}$transgene in the mushroom body (MB) could both rescue the LTM defect, demonstrating that the function of PTP10D in the mushroom body is sufficient for the physiological processes underlying LTM formation.

\section{Materials and Methods}

Fly stocks. $w^{1118}$ (isoCJ1) was used as a control in all of the experiments. The mutant named as chi by our laboratory was isolated in the screen for genes affecting long-term memory. $E P(X) 1172$ were a gift from Kai Zinn (California Institute of Technology, Pasadena, CA). The genetic backgrounds of all strains used were equilibrated to that of $w^{1118}$ (isoCJ1) by five generations of backcrossing, selecting for the presence of the $m w^{+}$ eye color marker carried within the PlacW transposon.

Pavlovian olfactory learning. The training and testing procedures were the same as described previously (Tully et al., 1994). During one training session, a group of $\sim 100$ flies was exposed sequentially to two odors (either octanol or methylcyclohexanol) for $60 \mathrm{~s}$ with a $45 \mathrm{~s}$ rest interval after each odor presentation. During exposure to the first odor, flies were simultaneously subjected to footshock (12 1.5-s pulses with $3.5 \mathrm{~s}$ intervals, $60 \mathrm{~V}$ ). To measure "immediate memory" (also referred to as "learning"), flies were transferred immediately after training to the choice point of a T maze and given a choice between the two odors for 2 min, after which they were trapped in their respective arms, anesthetized, and counted. A performance index (PI) was calculated from the distribution of flies in the T-maze. A PI of 0 represents a 50:50 distribution, whereas a PI of 100 represents $100 \%$ of flies avoiding the shock-paired odor by running into the other T-maze arm. Typically after one training session, memory retention in normal flies drops to near zero within $1 \mathrm{~d}$. To produce longer-lasting memory, flies were subjected to repetitive training sessions, either massed (10 sessions with no rest interval) or spaced (10 training sessions with a $15 \mathrm{~min}$ rest between each). After training, flies were transferred to food vials and stored at $18^{\circ} \mathrm{C}$ for $24 \mathrm{~h}$ before the T-maze test trial.

Olfactory acuity and shock reactivity. Odor-avoidance responses were quantified by exposing naive flies to each odor (octanol or methylcyclohexanol) versus air in the T maze for $120 \mathrm{~s}$, after which they were trapped in their respective arms, anesthetized, and counted. A PI was calculated for each odor individually as reported previously (Tully and Quinn, 1985). Shock-avoidance responses were quantified by inserting electrifiable grids into both arms of the T maze; shock pulses were delivered to one of the arms. After $60 \mathrm{~s}$, naive flies were trapped in their respective arms, anesthetized, and counted. Individual PIs were calculated as for odor acuity.

Statistic analyses. Data were analyzed with the program SPSS11 (SPSS, Chicago, IL). Overall ANOVA were followed by Student's $t$ test. To maintain an experiment-wise error rate of $\alpha=0.05$, the critical $p$ values for these individual comparisons were adjusted accordingly and are listed below for each experiment.

Heat-shock treatment. Flies were subjected to heat shock by placing them in empty vials in a $37^{\circ} \mathrm{C}$ incubator for $25 \mathrm{~min}$. Flies then were transferred back to bottles with food at room temperature (RT) (20$24^{\circ} \mathrm{C}$ ) for 3 or $27 \mathrm{~h}$ recovery periods.

Western blot analysis. Extracts were prepared from frozen whole flies as described previously (Yin et al., 1994). Protein concentrations were determined by the Bio-Rad (Hercules, CA) protein assay. Primary antibodies used were mouse monoclonal sera against PTP10D (1:100; kindly provided by Kai Zinn) and monoclonal antibody against $\beta$-actin (1: 20,000) (Sigma, St. Louis, MO). Products were visualized by enhanced chemiluminescence (Roche, Indianapolis, IN) and autoradiography.

Immunohistochemistry. Whole-mount embryos were dechorionated in $50 \%$ bleach for $5 \mathrm{~min}$, rinsed in water, and fixed for $30 \mathrm{~min}$ in heptane saturated with $4 \%$ formaldehyde in PBS (4\% formaldehyde in PBS/heptane at 1:3) at RT. Embryos then were devitellinized by shaking in heptane and $100 \%$ methanol for several seconds. After two rinses in methanol, embryos were washed four times in PBT (PBS, pH 7.2, and $0.2 \%$ Triton X-100). Embryos were preincubated in PBT with 5\% normal goat serum (block) for $1 \mathrm{~h}$ at RT. PTP10D monoclonal antibody was added to a final concentration of $2 \%$, and embryos were incubated overnight at $4^{\circ} \mathrm{C}$. Embryos were washed eight times with PBT for $1 \mathrm{~h}$ at RT. Horseradish peroxidase-conjugated anti-mouse secondary antibody was added and incubated for $45 \mathrm{~min}$ at RT. Embryos were washed eight times with PBS [containing (in mM) $2.7 \mathrm{KCl}, 4.3 \mathrm{Na}_{2} \mathrm{HPO}_{4}, 1.8 \mathrm{KH}_{2} \mathrm{PO}_{4}$, and 137 $\mathrm{NaCl}, \mathrm{pH} 7.2$ ] to completely remove Triton X-100; an equal volume of 1 $\mathrm{mg} / \mathrm{ml}$ diaminobenzidine with $0.3 \%$ hydrogen peroxide then was added to the wash and incubated for $3 \mathrm{~min}$ until the reaction product on embryos was visible. Finally, embryos were dehydrated in ethanol and xylene and mounted in Araldite.

Immunohistochemistry on adult heads was performed on paraffin sections. For the immunohistochemical screen of viable mutants, five control flies and five PTP10D mutants were mounted side by side in a fly collar and sectioned.

For paraffin sections, the flies were fixed in Carnoy's fixative $(60 \%$ ethanol, $30 \%$ chloroform, and $10 \%$ acetic acid) for $4 \mathrm{~h}$ at RT, washed two times in ethanol for $30 \mathrm{~min}$ each, washed in absolute ethanol (dehydrated) for $1 \mathrm{~h}$, treated with methylbenzoate overnight, and embedded in paraffin. We obtained $7 \mu \mathrm{m}$ sections, deparaffinized them in a xylene bath for 75 min, rehydrated them through $100-25 \%$ ethanol series and distilled water, blocked the tissue with goat serum in PBT for $40 \mathrm{~min}$ at $\mathrm{RT}$, and then incubated it with a 1:50 dilution of anti-PTP10D monoclonal antibody overnight at $4^{\circ} \mathrm{C}$. Sections were washed in PBT at least three times and incubated in HRP-conjugated anti-mouse secondary antibody for $1 \mathrm{~h}$ at RT. Finally, sections were washed in PBS at least three times and stained by DAB kit at RT.

Reverse transcription-PCR. For reverse transcription-PCR analysis of PTP10D, total RNA was prepared from control flies $\left[w^{1118}\right.$ (isoCJ1)] or chi mutants using the Qiagen (Valencia, CA) kit. RNA was quantified with a spectrophotometer, and equal amounts of RNA from the two preparations were reverse transcribed and subjected to PCR analysis using the protocols of ImProm-II Reverse Transcription System provided

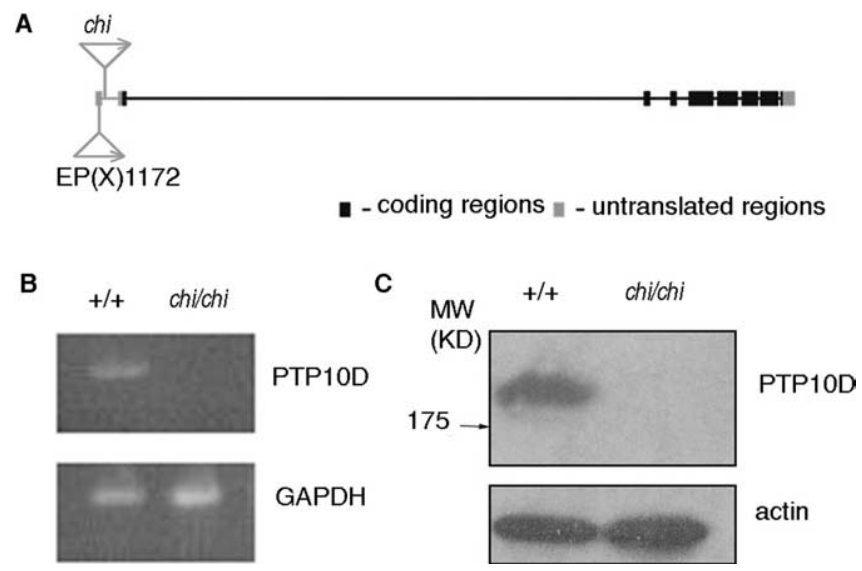

Figure 1. chi mutants carry a P element inserted in the PTP10D gene, reducing levels of PTP10D mRNA and protein. $A$, The intron/exon structure of PTP10D is shown, along with the PlacW insertion in chi mutants and a second independently derived Pinsertion [EP(X)1172]. The directions of enhancer-driven transcription within each P element are indicated by the arrow. $\boldsymbol{B}$, Reverse transcription-PCR analysis of total RNA from control flies $\left[W^{1118}\right.$ (isoCl1)] and the chi mutant. mRNA expression of PTP10D is significantly lower in mutant than in control flies. In contrast, mRNA expression of a control gene, GAPDH, is similar between mutant and control flies, indicating that similar total amounts of RNA were loaded into each lane. C, Western blot analysis of PTP10D protein expression from control [ $w^{1118}$ (isoCl1)] flies and chi mutants. A band of the appropriate size [molecular weight (MW) of $175 \mathrm{kDa}$ ] was detected in control flies but was not in chi/chi mutants. In contrast, expression of an actin loading control was similar between control and mutant flies. 
by Promega (Madison, WI). The resulting cDNA was divided into two equal parts, which were used as templates in PCR amplifications of PTP10D and glyceraldehyde-3-phosphate dehydrogenase (GAPDH) (loading control), respectively. The PTP10D primers used were $5^{\prime}$-ggcccagaatatgaccaccaggaa-3' and $5^{\prime}$ ggcgctaatatcggcatcgtttga- $3^{\prime}$, and the GAPDH primers were $5^{\prime}$-ccactgccgaggaggtcaacta- $3^{\prime}$ and $5^{\prime}$-gctcagggtgattgcgtatgca-3'.

Plasmid rescue. Genomic DNA flanking the chi $\mathrm{P}$ element insertion site was obtained by digesting total genomic DNA with EcoRI or SacII, circularizing the resulting fragments and transforming bacteria to isolate DNA fragments containing an amp resistance gene within the PlacW transposon. The cloned fragments then were sequenced using standard methods.

Generation of hs- and UAS-PTP10D ${ }^{+}$transgenic flies. Plasmid re52018 (Berkeley Drosophila Genome Project, Berkeley, CA) contained a full-length cDNA fragment for PTP10D within the pFLC-1 vector. This cDNA fragment extends from $432 \mathrm{bp}$ upstream of the putative translation start codon to 2983 bp downstream of the stop codon. The re52018 plasmid and Pcasper-hs vector were restriction digested with $K p n I$ and StuI, respectively. The liberated PTP10 cDNA insert and linearized Pcasper-hs vector then were blunt ended using T4 polymerase, subsequently cut by the enzyme NotI, recovered, and then joined together by T4 ligase at $4^{\circ} \mathrm{C}$ for $>24 \mathrm{~h}$. Finally, bacteria were transformed. Sequencing of colony isolates confirmed the cloning of the hs-PTP10D ${ }^{+}$ construct. Germ-line transformation was accomplished by injecting the hs-PTP10D ${ }^{+}$constructs into control embryos using standard techniques (Rubin and Spradling, 1982; Spradling and Rubin, 1982). One transposant was recovered, with the hs-PTP10D ${ }^{+}$transgene inserted somewhere on the second chromosome. The genetic background of this transgenic line was equilibrated to that of $w^{1118}$ (isoCJ1) flies via five generations of backcrossing. For rescue of mutant memory, chi/chi; hs$P T P 10 D^{+} / h s-P T P 10 D^{+}$flies were bred.

A 7311 bp NotI-KpnI fragment, restriction digested from re52018, was cloned into the PUAST vector similarly cut with NotI and KpnI. After germ-line transformation as above, one transposant line was recovered with the UAS-PTP10D ${ }^{+}$transgene inserted somewhere on the third chromosome. Here again, the genetic background of this strain was equilibrated to that of $w^{1118}$ (isoCJ1) flies via five generations of backcrossing.

\section{Results}

\section{Mutations of PTP10D disrupt LTM formation}

We performed a P element insertional mutagenesis to screen for $\mathrm{X}$-linked mutants that disrupt $1 \mathrm{~d}$ memory after spaced training. Plasmid rescue of genomic DNA flanking the P element (PlacW) insertion in one identified mutant, which we named chi ("chi" is a Chinese word that can be translated as "foolish"), revealed the transposon to be inserted in the first intron of PTP10D, $345 \mathrm{bp}$ downstream of the splice donor site of exon 1 and 943 bp upstream of the start codon in exon 2 (Fig. 1A). Reverse transcription-PCR analyses revealed that expression of the PTP10D transcript is greatly reduced in chi mutants (Fig. $1 B$ ). Western blot analysis identified a single protein band of $190 \mathrm{kDa}$ in control flies, which is consistent with a previous observation (Tian et al., 1991), and this band was undetected in chi/chi mutants (Fig. 1C). These molecular results suggest that PTP10D is disrupted in chi mutants.

Our studies also used another, independently isolated mutation of PTP10D, EP(X)1172, which also results from a P element insertion within the first exon (Fig. 1A). Homozygous
$\mathrm{EP}(\mathrm{X}) 1172$ mutants also were reported to express very low levels of PTP10D protein (Tian et al., 1991; Sun et al., 2000).

These PTP10D mutants were found to be defective in $1 \mathrm{~d}$ memory after spaced training. Both chi/chi and $\operatorname{EP}(\mathrm{X}) 1172 /$ $\mathrm{EP}(\mathrm{X}) 1172$ homozygous mutants showed significantly lower $1 \mathrm{~d}$ memory after spaced training than control flies (Fig. 2 A). More importantly, the two mutant alleles failed to complement; $1 \mathrm{~d}$ memory after spaced training in chi/EP(X)1172 heteroallelic mutants was significantly lower than control flies (Fig. $2 B$ ). This finding demonstrates that loss of function of the PTP10D gene per se is responsible for the defect in $1 \mathrm{~d}$ memory after spaced training.

To determine which memory phase was disrupted in PTP10D mutants, we first examined $1 \mathrm{~d}$ memory after massed training and found it statistically indistinguishable from control for both chi/ chi and $\operatorname{EP}(\mathrm{X}) 1172 / \mathrm{EP}(\mathrm{X}) 1172$ mutants (Fig. 2A). This observation demonstrates that ARM is normal in PTP10D mutants. Next, we examined memory immediately or $3 \mathrm{~h}$ after one training session, which assess STM or MTM, respectively. For both aspects of memory retention, performance was statistically indistinguishable from control in chi/chi mutants (Fig. 2C). Together, these results establish that STM, MTM, and ARM are normal, but LTM specifically is disrupted, in PTP10D mutants.

For immediate and $3 \mathrm{~h}$ memory after one training session and for $1 \mathrm{~d}$ memory after massed training, performance is indistinguishable from control, suggesting that the mutants are able to sense and react to odors and footshock normally. To eliminate formally the possibility that these sensorimotor responses are defective, we examined olfactory acuity and shock reactivity in PTP10D mutants. In both assays, performance is statistically indistinguishable from control in chi/chi and $\operatorname{EP}(\mathrm{X}) 1172 /$ EP(X)1172 mutants (supplemental Table 1, available at www. jneurosci.org as supplemental material). These results further specify the behavioral defect in PTP10D mutants to be restricted to LTM.

\section{PTP10D is required during memory formation, not during development or maintenance}

The chi and $\mathrm{EP}(\mathrm{X}) 1172$ mutations disrupt PTP10D function throughout development. Thus, the specific defect in LTM might nonetheless derive from abnormal development of the neural 
A $+/+$ chi/chi;hs-PTP10D/hs-PTP10D ${ }^{+}$ Ohr Ohr $3 \mathrm{hr} 27 \mathrm{hr}$
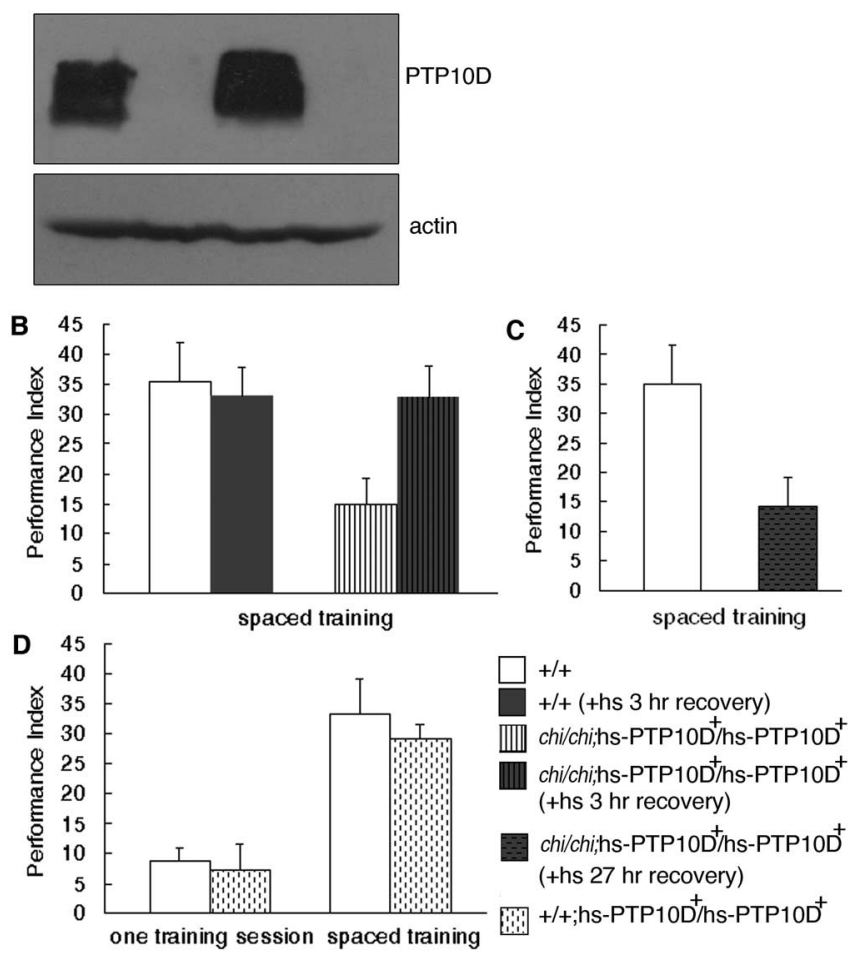

Figure 3. The memory defect of PTP10D mutants can be rescued by acute induction of hs-PTP10D ${ }^{+}$. $A$, Western blot analysis of PTP10D protein levels in control flies and in chi/chi; hs-PTP10D ${ }^{+} /$hs-PTP10D ${ }^{+}$transgenic mutants without heat shock and in chi/chi;hs-PTP10D ${ }^{+} /$ hs-PTP10D ${ }^{+}$transgenic mutants 3 and $27 \mathrm{~h}$ after heat shock (from 25 to $37^{\circ} \mathrm{C}$ for $25 \mathrm{~min}$ ). $\boldsymbol{B}$, Control flies $\left[w^{1118}\right.$ (isoCJ1)] or chi/chi;hs-PTP10D ${ }^{+} /$hs-PTP10D $^{+}$transgenic mutants were subjected to spaced training without $(-\mathrm{hs})$ or $3 \mathrm{~h}$ after $(+\mathrm{hs})$ heat shock treatment. ANOVA revealed a significant difference of genotypes $\left(F_{(3,28)}=25.4 ; p<0.001\right)$. In the absence of heat shock, $1 \mathrm{~d}$ memory was significantly lower than normal in transgenic mutants $(p<0.001)$. After heat shock treatment, however, $1 \mathrm{~d}$ memory was normal in transgenic mutants ( $p=$ 0.94). Heat shock itself also did not produce any nonspecific effect on $1 \mathrm{~d}$ memory in control flies $(p=0.52) . n=8 \mathrm{PIs}$ per group. $\mathbf{C}$, One day memory after spaced training in control flies is significantly higher than in chi/chi;hs-PTP10D ${ }^{+} /$hs-PTP10D ${ }^{+}$transgenic mutants when trained $27 \mathrm{~h}$ after heat shock (from 25 to $37^{\circ} \mathrm{C}$ for $\left.25 \mathrm{~min}\right)\left(F_{(1,14)}=46.2 ; p<0.001\right) . n=8 \mathrm{Pls}$ per group. $D, 0$ ne day memory is not affected by overexpression of hs-PTP10D + in control flies. Flies were subjected to the same heat shock treatment as described in Results. After a $3 \mathrm{~h}$ recovery from heat shock, flies received either one training session or spaced training, and $1 \mathrm{~d}$ memory retention was quantified. Mean Pls of control $(+/+)$ and hs-PTP10D ${ }^{+}$transgenic flies $\left(+/+;\right.$ hs-PTP10D ${ }^{+} /$hs-PTP10D $\left.^{+}\right)$were similar after one training session $\left(F_{(1,10)}=\right.$ $0.51 ; p=0.49)$ or after spaced training $\left(F_{(1,10)}=1.52 ; p=0.25\right) . n=6$ Pls for each group.

structures that subserve LTM. To evaluate this possibility, we constructed chi/chi;hs-PTP10D ${ }^{+} /$hs $^{-P T P 10 D}{ }^{+}$transgenic mutants, which carry a full-length cDNA of PTP10D under the control of the $h s p 70$ heat shock promoter. Western blot analysis revealed that expression levels of transgenic PTP10D protein were undetected in adult extracts from chi/chi;hs-PTP10D ${ }^{+} /$hs $^{-}$ PTP10D ${ }^{+}$transgenic mutants at $25^{\circ} \mathrm{C}$ (Fig. $3 A$ ) and $18^{\circ} \mathrm{C}$ (data not shown), whereas PTP10D protein was detected in such transgenic mutants $3 \mathrm{~h}$ after a 25 min heat shock (from 25 to $37^{\circ} \mathrm{C}$ ) (Fig. 3A). Concomitant with these observations, $1 \mathrm{~d}$ memory after spaced training was defective in transgenic mutants at $25^{\circ} \mathrm{C}$ but was statistically indistinguishable from control when animals were trained $3 \mathrm{~h}$ after the heat shock treatment (Fig. $3 B$ ). This rescue effect cannot be attributed to altered sensorimotor responses, because control flies and transgenic mutants showed indistinguishable olfactory acuity and shock reactivity before or after heat shock (supplemental Table 1, available at www. jneurosci.org as supplemental material). Thus, acutely induced expression of an hs-PTP10D ${ }^{+}$transgene in the adult is sufficient to rescue the LTM defect of chi mutants.

We further explore the acute role of PTP10D in LTM. Western blot analysis revealed that heat shock-induced PTP10D protein expression in transgenic mutants decreased to undetectable levels within $27 \mathrm{~h}$ after heat shock treatment (Fig. 3A). Accordingly, $1 \mathrm{~d}$ memory after spaced training again was lower than control flies when animals were trained $27 \mathrm{~h}$ after heat shock (Fig. $3 C$ ). In contrast, LTM was rescued in the transgenic mutants if trained $3 \mathrm{~h}$ after heat shock but tested $27 \mathrm{~h}$ later even at which acutely expressed PTP10D was becoming undetectable.

To assess any potential enhancement of memory formation, we also evaluated the effects of overexpression of PTP10D in the wild-type background. Transgenic flies (hs-PTP10D ${ }^{+}$) were subjected a 25 min heat shock (from 25 to $37^{\circ} \mathrm{C}$ ) and trained $3 \mathrm{~h}$ afterward. Overexpression of hs-PTP10D ${ }^{+}$did not affect $1 \mathrm{~d}$ memory after one training session or after spaced training (Fig. 3D).

Together, these data suggest that PTP10D plays an acute role that is essential for LTM formation but not for the maintenance of LTM, and any possible subtle developmental defects can be overcome with expression of PTP10D in adult flies.

\section{PTP10D is widely expressed in the embryonic CNS and in the adult brain}

Consistent with previous reports (Tian et al., 1991; Yang et al., 1991), our immunohistochemical analysis revealed that PTP10D protein is expressed in the ventral nerve cord of embryonic stage 14 control flies. Such immunostaining was not detected in $\mathrm{chi} / \mathrm{chi}$ mutant embryos (Fig. 4A). Immunohistochemical analysis of PTP10D protein expression in adult brain was done on paraffin sections, using a monoclonal antibody. In control flies, antigen was detected throughout the neuropillar regions of the brain, including the mushroom bodies, the central complex (CC), and the antennal lobes (Fig. $4 B-D$ ). In chi/chi mutants, antigen immunoreactivity was greatly reduced (Fig. $4 E$ ). Using hematoxylin and eosin staining of paraffin sections, no gross structural defects could be seen in chi/chi mutant adult brains (supplemental Fig. S1, available at www.jneurosci.org as supplemental material).

\section{Expression of PTP10D ${ }^{+}$in mushroom body is sufficient to rescue the memory defect of chi mutants}

The expression pattern of PTP10D certainly did not provide any clues as to where in the brain its function is required for LTM formation. Two logical possibilities based on several previous studies are the MBs and the CC (Connolly et al., 1996; Zars et al., 2000; Dubnau et al., 2001; Pascual and Preat, 2001; Isabel et al., 2004; McGuire et al., 2005; Liu et al., 2006). To evaluate any putative roles for these neuroanatomical sites, we constructed

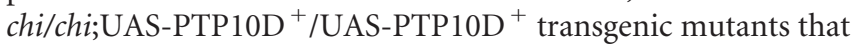
carry a full-length cDNA for PTP10D under the control of a UAS promoter sequence, which binds the yeast galactosidase-4 (Gal4) transcription factor (Brand and Perrimon, 1993). These transgenic mutants are defective in $1 \mathrm{~d}$ memory after spaced training (Fig. 5). Virgin transgenic mutants were bred to males of either the OK107 Gal4 enhancer trap line, which drives UAS-transgene expression in MBs (and pars intercerebralis) or the c232 enhancer trap line, which drives UAS-transgene expression in the central complex. Male progeny from these crosses then were subjected to spaced training. One day memory for $c h i / Y ;+/+$;UAS$\mathrm{PTP}_{10 \mathrm{D}}{ }^{+} /+; \mathrm{OK} 107 /+$ flies was statistically indistinguishable 
from control, whereas that for chi/Y;c232/ + ;UAS-PTP10D ${ }^{+} /+;+/+$flies was significantly reduced and indistinguishable from chi/chi;UAS-PTP10D ${ }^{+} / \mathrm{UAS}$ $\mathrm{PTP} 10 \mathrm{D}^{+}$transgenic mutants (Fig. 5). Virgin transgenic mutants were also bred to males of c309 MB driver in the mutant chi background. All progeny from this cross then were subjected to spaced training. One day memory for chi/chi;c309/+; UAS-PTP10D/+ also was statistically indistinguishable from control (Fig. 5). Here again, sensorimotor responses to odors and footshock were statistically indistinguishable from control (supplemental Table 1, available at www.jneurosci.org as supplemental material). Together, these results demonstrate that transgenic PTP10D function in MBs is sufficient to rescue the defective LTM of chi mutants.

\section{Discussion}

From a behavioral screen for long-term memory mutants, we identified $c h i$, which carries a molecular lesion of the PTP10D gene. The chi mutation derives from a $\mathrm{P}$ element insertion into the first intron of PTP10D, leading to drastic reductions in mRNA and protein from this transcription unit (Fig. 1). Homozygotes of another, independently isolated mutation in PTP10D, $E P(X) 1172$, also are defective for long-term memory, and the two alleles fail to complement each other for this phenotype (Fig. 2). Assays for different memory phases reveal the memory defect of chi mutants to be specific for long-term memory; STM, MTM, and ARM all were statistically indistinguishable from controls (Fig. 2), as were basic sensorimotor responses to odors and footshock (supplemental Table 1, available at www. jneurosci.org as supplemental material). These observations confirm that mutations in the chi locus lead to specific LTM defects.

This defect is not resulted from developmental abnormality for the LTM phenotype of chi mutants and can be rescued by acutely induced expression of an hs-PTP10D ${ }^{+}$transgene in the adult fly (Fig. 3), a conclusion also supported by the lack of abnormalities in gross brain morphology in chi mutants (supplemental Fig. S1, available at www.jneurosci.org as supplemental material). More specifically, the Chi protein PTP10D plays an essential acute role in formation of LTM but is not required for maintenance of LTM. We showed that this rescue occurred only when training took place within $3 \mathrm{~h}$ of heat shock when induced expression of PTP10D was abundant. However, the heat shock induction failed to rescue LTM when training was conducted $27 \mathrm{~h}$ later at which induced expression of PTP10D was becoming undetectable. Moreover, at testing of memory $(27 \mathrm{~h}$ after heat shock), although induced expression of PTP10D was undetectable, LTM remained to be rescued if training happened within an interval during which induced expression of PTP10D was abundant. Such observations provide a strong demonstration of an acute role for PTP10D during LTM formation.
Finally, function of PTP10D for memory formation is localized in the MB. Transgenic expression of a UAS-PTP10D ${ }^{+}$transgene only in MBs is sufficient to rescue the LTM defect of chi mutants (Fig. 5), a result consistent with many other studies that support a role for MBs during olfactory memory formation (Connolly et al., 1996; Zars et al., 2000; Dubnau et al., 2001; McGuire et al., 2001; Presente et al., 2004). In particular, it has been shown that the absence of the vertical lobes of the mushroom body disrupts LTM (Pascual and Preat, 2001; Isabel et al., 2004), and mutations in Crammer peptides that are expressed in the glial cells around the mushroom body also lead to defective LTM (Comas et al., 2004). This study presents for the first time that the function of a protein in the mushroom body is required for formation of LTM. All of these lines of evidence lead to the conclusion that this receptor-like tyrosine phosphatase plays a critical role in biochemical cascades that mediate long-term memory formation in Drosophila.

Several PTPs have been examined in Drosophila. PTP69D plays a role in axon guidance, whereas Drosophila leukocyte common antigen-related Dlar appears to be required for maturation of growth cones in synapses (Desai et al., 1996; Krueger et al., 

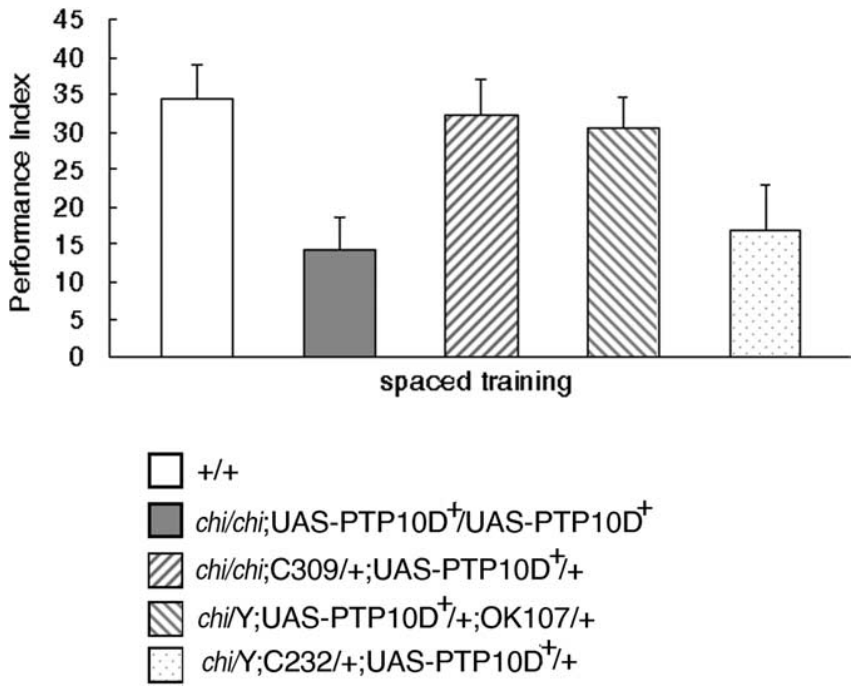

Figure 5. The LTM defect of chi mutants can be rescued by expression of PTP10D ${ }^{+}$in MBs but not in CC. One day memory after spaced training was quantified in different groups of control flies [ $w^{1118}$ (isoCJ1)], chi/chi;UAS-PTP10D ${ }^{+} /$UAS-PTP10D ${ }^{+}$transgenic mutants, chi/Y; $+/+;$ UAS-PTP10D ${ }^{+} /+; 0 \mathrm{OK107/+}$ flies, or chi/Y;(232/+;UAS-PTP10D ${ }^{+} /+;+/+$flies. ANOVA revealed a significant difference of genotypes $\left(F_{(4,55)}=23.3 ; p<0.001\right)$. Mean PIs were significantly lower than normal in chi/chi;UAS-PTP10D ${ }^{+} /$UAS-PTP10D ${ }^{+}$transgenic mutants $(p<0.001)$ and in chi $/ Y ;\left(232 /+;\right.$ UAS-PTP10D ${ }^{+} /+;+/+$flies $(p<0.001)$. The mean Pls of chi/chi;UAS-PTP10D ${ }^{+} /$UAS-PTP10D $^{+}$and chi/Y;C232/+;UAS-PTP10D ${ }^{+} /+$; $+/+$ are significantly lower than that of chi/Y; $+/+;$ UAS-PTP10D ${ }^{+} /+; 0 \mathrm{KK} 107 /{ }^{+}$. (both $p<$ 0.001), and the mean Pls of chi/chi;UAS-PTP10D ${ }^{+} /$UAS-PTP10D ${ }^{+}$and chi/Y;(232/+;UASPTP10D ${ }^{+} /+;+/+$are significantly lower than that of chi/chi;C309/+;UAS-PTP10D ${ }^{+} /+$ flies $(p<0.001$ and $p=0.002$, respectively). In contrast, the mean PI of chi/Y; $+/+; U A S-$ PTP10D ${ }^{+} /+; 0 K 107 / /^{+}$flies and chi/chi;C309/+;UAS-PTP10D ${ }^{+} /+$flies were similar to that of controls ( $p=0.051$ and $p=0.50$, respectively). $n=12$ for each genotype.

1996; Sun et al., 2000). Our data show that PTP10D is expressed widely in embryonic and adult CNS. Thus, one might expect PTP10D also to play a role in neurodevelopment. To that end, the axon guidance defect of PTP69D mutants is exacerbated in PTP69D;PTP10D double mutants (Sun et al., 2000), but PTP10D single mutants display no observable defects in development of the CNS (Desai et al., 1996; Sun et al., 2000). Our data support these observations and suggest, instead, a more important role for PTP10D in the biochemistry of memory formation.

Our study provides the following insights. Primarily, this is a novel report in invertebrates that a receptor-like tyrosine phosphatase is involved in memory formation. In vertebrates, there are a number of reports that suggest an involvement of receptorlike tyrosine phosphatases in learning and memory, although it remains to be determined whether such effects are specific to long-term memory formation (Uetani et al., 2000; Skelton et al., 2003; Kolkman et al., 2004). In any case, these observations indicate that signaling via receptor-like tyrosine phosphatases may be a conserved biochemical pathway for learning and memory, as for the cAMP pathway. Second, the extracellular structure of PTP10D is similar to other cell adhesion molecules, suggesting that cell-cell interaction might be a critical signal transduction mechanism during memory formation. Consistent with this notion, mutants for Drosophila integrin and Fas II have been reported to disrupt STM (Grotewiel et al., 1998; Cheng et al., 2001). More generally, cell adhesion molecules have been implicated widely for synaptic plasticity in vertebrates and invertebrates (for review, see Murase and Schuman, 1999).

\section{References}

Brand AH, Perrimon N (1993) Targeted gene expression as a means of altering cell fates and generating dominant phenotypes. Development 118:401-415.

Cheng Y, Endo K, Wu K, Rodan AR, Heberlein U, Davis RL (2001) Drosophila fasciclinII is required for the formation of odor memories and for normal sensitivity to alcohol. Cell 105:757-768.

Comas D, Petit F, Preat T (2004) Drosophila long-term memory formation involves regulation of cathepsin activity. Nature 430:460-463.

Connolly JB, Roberts IJ, Armstrong JD, Kaiser K, Forte M, Tully T, O’Kane CJ (1996) Associative learning disrupted by impaired Gs signaling in Drosophila mushroom bodies. Science 274:2104-2107.

Desai CJ, Gindhart Jr JG, Goldstein LS, Zinn K (1996) Receptor tyrosine phosphatases are required for motor axon guidance in the Drosophila embryo. Cell 84:599-609.

Dubnau J, Grady L, Kitamoto T, Tully T (2001) Disruption of neurotransmission in Drosophila mushroom body blocks retrieval but not acquisition of memory. Nature 411:476-480.

Grotewiel MS, Beck CD, Wu KH, Zhu XR, Davis RL (1998) Integrinmediated short-term memory in Drosophila. Nature 391:455-460.

Isabel G, Pascual A, Preat T (2004) Exclusive consolidated memory phases in Drosophila. Science 304:1024-1027.

Kolkman MJ, Streijger F, Linkels M, Bloemen M, Heeren DJ, Hendriks WJ, Van der Zee CE (2004) Mice lacking leukocyte common antigen-related (LAR) protein tyrosine phosphatase domains demonstrate spatial learning impairment in the two-trial water maze and hyperactivity in multiple behavioural tests. Behav Brain Res 154:171-182.

Krueger NX, Van Vactor D, Wan HI, Gelbart WM, Goodman CS, Saito H (1996) The transmembrane tyrosine phosphatase DLAR controls motor axon guidance in Drosophila. Cell 84:611-622.

Liu G, Seiler H, Wen A, Zars T, Ito K, Wolf R, Heisenberg M, Liu L (2006) Distinct memory traces for two visual features in the Drosophila brain. Nature 439:551-556.

McGuire SE, Le PT, Davis RL (2001) The role of Drosophila mushroom body signaling in olfactory memory. Science 293:1330-1333.

McGuire SE, Deshazer M, Davis RL (2005) Thirty years of olfactory learning and memory research in Drosophila melanogaster. Prog Neurobiol 76:328-347.

Murase S, Schuman EM (1999) The role of cell adhesion molecules in synaptic plasticity and memory. Curr Opin Cell Biol 11:549-553.

Neel BG, Tonks NK (1997) Protein tyrosine phosphatases in signal transduction. Curr Opin Cell Biol 9:193-204.

Pascual A, Preat T (2001) Localization of long-term memory within the Drosophila mushroom body. Science 294:1115-1117.

Presente A, Boyles RS, Serway CN, de Belle JS, Andres AJ (2004) Notch is required for long-term memory in Drosophila. Proc Natl Acad Sci USA 101:1764-1768.

Purcell AL, Carew TJ (2003) Tyrosine kinases, synaptic plasticity and memory: insights from vertebrates and invertebrates. Trends Neurosci 26:625-630.

Rubin GM, Spradling AC (1982) Genetic transformation of Drosophila with transposable element vectors. Science 218:348-353.

Schindelholz B, Knirr M, Warrior R, Zinn K (2001) Regulation of CNS and motor axon guidance in Drosophila by the receptor tyrosine phosphatase DPTP52F. Development 128:4371-4382.

Skelton MR, Ponniah S, Wang DZ, Doetschman T, Vorhees CV, Pallen CJ (2003) Protein tyrosine phosphatase alpha (PTP alpha) knockout mice show deficits in Morris water maze learning, decreased locomotor activity, and decreases in anxiety. Brain Res 984:1-10.

Spradling AC, Rubin GM (1982) Transposition of cloned P elements into Drosophila germ line chromosomes. Science 218:341-347.

Sun Q, Bahri S, Schmid A, Chia W, Zinn K (2000) Receptor tyrosine phosphatases regulate axon guidance across the midline of the Drosophila embryo. Development 127:801-812.

Tian SS, Tsoulfas P, Zinn K (1991) Three receptor-linked protein-tyrosine phosphatases are selectively expressed on central nervous system axons in the Drosophila embryo. Cell 67:675-680.

Tully T, Quinn WG (1985) Classical conditioning and retention in normal and mutant Drosophila melanogaster. J Comp Physiol A Neuroethol Sens Neural Behav Physiol 157:263-277. 
Tully T, Boynton S, Brandes C, Dura JM, Mihalek R, Preat T, Villella A (1990) Genetic dissection of memory formation in Drosophila melanogaster. Cold Spring Harb Symp Quant Biol 55:203-211.

Tully T, Preat T, Boynton SC, Del Vecchio M (1994) Genetic dissection of consolidated memory in Drosophila. Cell 79:35-47.

Uetani N, Kato K, Ogura H, Mizuno K, Kawano K, Mikoshiba K, Yakura H, Asano M, Iwakura Y (2000) Impaired learning with enhanced hippocampal long-term potentiation in PTPdelta-deficient mice. EMBO J 19:2775-2785.

Van Vactor D, O’Reilly AM, Neel BG (1998) Genetic analysis of protein tyrosine phosphatases. Curr Opin Genet Dev 8:112-126.

Wallace MJ, Batt J, Fladd CA, Henderson JT, Skarnes W, Rotin D (1999)
Neuronal defects and posterior pituitary hypoplasia in mice lacking the receptor tyrosine phosphatase PTPsigma. Nat Genet $21: 334-338$

Yang XH, Seow KT, Bahri SM, Oon SH, Chia W (1991) Two Drosophila receptor-like tyrosine phosphatase genes are expressed in a subset of developing axons and pioneer neurons in the embryonic CNS. Cell 67:661-673.

Yin JC, Wallach JS, Del Vecchio M, Wilder EL, Zhou H, Quinn WG, Tully T (1994) Induction of a dominant negative CREB transgene specifically blocks long-term memory in Drosophila. Cell 79:49-58.

Zars T, Fischer M, Schulz R, Heisenberg M (2000) Localization of a shortterm memory in Drosophila. Science 288:672-675. 